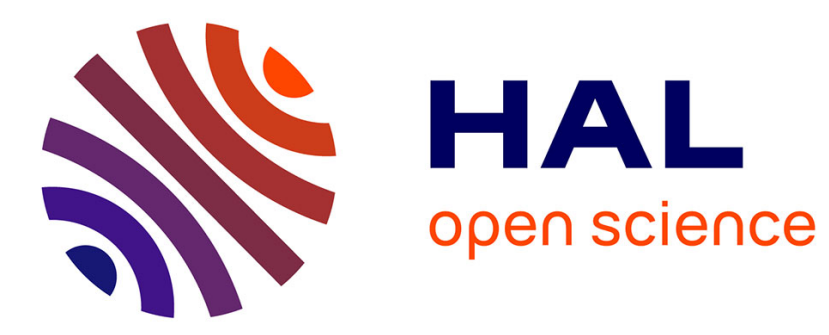

\title{
Global analysis of a continuous-time flow which computes time-optimal switchings
}

Frédéric Grognard, Rodolphe Sepulchre

\section{To cite this version:}

Frédéric Grognard, Rodolphe Sepulchre. Global analysis of a continuous-time flow which computes time-optimal switchings. 40th IEEE Conference on Decision and Control, Dec 2001, Orlando, United States. pp.3826-3831, 10.1109/.2001.980460 . hal-01091651

\section{HAL Id: hal-01091651 \\ https://hal.inria.fr/hal-01091651}

Submitted on 5 Dec 2014

HAL is a multi-disciplinary open access archive for the deposit and dissemination of scientific research documents, whether they are published or not. The documents may come from teaching and research institutions in France or abroad, or from public or private research centers.
L'archive ouverte pluridisciplinaire HAL, est destinée au dépôt et à la diffusion de documents scientifiques de niveau recherche, publiés ou non, émanant des établissements d'enseignement et de recherche français ou étrangers, des laboratoires publics ou privés. 


\title{
Global analysis of a continuous-time flow which computes time-optimal switchings
}

\author{
F. Grognard ${ }^{\dagger}$, R. Sepulchre ${ }^{\ddagger \dagger}$, \\ $\dagger$ Center for Systems Engineering and Applied Mechanics \\ Université catholique de Louvain, \\ Av. G. Lemaitre 4, B1348 Louvain-La-Neuve, Belgium. \\ $\$$ Institut Montefiore, B28 \\ Université de Liège, \\ B4000 Liège Sart-Tilman, Belgium. \\ email: $\{$ grognard $\},\{$ sepulchre $\} @$ montefiore.ulg.ac.be
}

\begin{abstract}
The minimum-time bounded control of linear systems is generically bang-bang and the number of switchings does not exceed the dimension of the system if the eigenvalues of the system matrix are real. This paper proposes a synthesis method for such problems based on dynamical systems that 'compute' the optimal sequence of switching times.
\end{abstract}

\section{Introduction}

This paper addresses the classical synthesis problem of time-optimal control laws for a single-input linear system $\dot{z}=A z+b v$ subject to the bounded input constraint $|v| \leq 1$. We consider the situation of a controllable pair $(A, b)$ and assume that all the eigenvalues of $A$ are real. The computation of the time-optimal control reduces to the computation of the optimal sequence of switchings times $0=t_{0} \leq t_{1} \leq \ldots \leq t_{n}=T$ of the optimal bang-bang control, or equivalently, the optimal sequence of time-intervals $\bar{x}_{1}=t_{1}-t_{0}, \bar{x}_{2}=$ $t_{2}-t_{1}, \ldots, \bar{x}_{n}=t_{n}-t_{n-1}$.

In this paper, we construct continuous time-systems $\dot{x}=f(x)$ which 'produce' an optimal sequence $\bar{x}=$ $\left(\bar{x}_{1}, \ldots, \bar{x}_{n}\right)^{T}$, in the sense that their equilibrium set coincides with the set of optimal sequences (a singleton if $z_{0}$ is not on a switching surface) and that it is a stable attractor with an arbitrarily large region of attraction. The systems are positive, i.e. they are only defined in the open positive orthant $\mathcal{O}_{n}^{+}=\left\{x \mid x_{i}>0, i=1, \cdots, n\right\}$ and the dynamics leave this open set invariant. Our main result shows that, under proper time-scale decomposition, the convergence of solutions to the desired equilibrium $x=\bar{x}$ can be enforced in a rather transparent way, through a sequence of nested invariant manifolds. This manifold structure connects the convergence properties of the algorithm to the geometry of the time-optimal control problem and supports the excellent convergent properties that are observed in simulations, even when the time scales are no longer enforced.

The algorithms discussed in the present paper are based on a particular heuristics, to be explained in Section 2. In that respect, they differ from the realm of gradient-based iterative methods for the synthesis of time-optimal controllers, that date back almost to the starting point of the time-optimal control problem itself $[1,3,5,6,9,12]$, and which are, in general, sensitive to the starting condition (initial guess) and have poor convergence properties. This is in contrast with the convergence properties displayed in the present paper.

A 'modern' motivation for studying iterative methods for the synthesis of optimal control laws is in their potential implementation in a receding-horizon fashion, so as to make corrections to the control policy when the plant is affected by inaccuracies or by external disturbances. With the current computing power and with the maturity reached by the theory of receding-horizon control [11], there is a renewed interest in efficient algorithms for the synthesis of optimal control laws.

The remainder of the paper is organized as follows. In Section 2 we provide some background and the heuristics of the iterative methods discussed in this paper. In Section 3, the main features of the algorithm and some of its convergence issues are illustrated on an example. In Sections 4 and 5, the time-scales separation and the convergence of the fast and slow subsystem of a particular continuous-time version of the algorithm are analyzed. Section 6 puts those analyses together to obtain a semiglobal convergence result. Concluding remarks are presented in Section 7. 


\section{Heuristics for iterative computation of optimal switching times}

Consider the linear system

$$
\dot{z}=A z+b v
$$

where $z \in \mathbb{R}^{n}$ is the state and $v \in \mathbb{R}$ is the input which is constrained to $|v| \leq 1$. We assume that the pair $(A, b)$ is controllable see. We further assume that the eigenvalues of matrix $A$ are real. The problem is to find the control function $v^{0}(t)$ that steers the trajectory from a specified initial state $z(0)=z_{0}$ to a specified final state here chosen to be the origin $z\left(t_{f}\right)=z_{f}=0$ in minimum time $t_{f}^{0}$. We assume that $z_{0}$ is in the nullcontrollable set, i.e. the time-optimal problem has a solution. From the maximum principle [10], the timeoptimal control is 'bang-bang' (i.e., piecewise constant, with each component assuming only the values \pm 1 ) and it has at most $n-1$ switchings; and, any 'bangbang' policy with at most $n-1$ switchings is optimal in the sense that whatever state the plant reaches using such a control, it does so in optimal time.

As a consequence of these properties, the search for the optimal control can be restricted to the steering controls that are defined by a sequence of $n$ time intervals $x_{i} \triangleq t_{i}-t_{i-1}$ and the corresponding sequence of constant control values $u_{i}$. This class of piecewise constant controls (see Figure 1) is characterized by a pair of vectors $(x, u)$, where $x$ denotes the vector of time intervals and $u$ denotes the vector of control values. From the
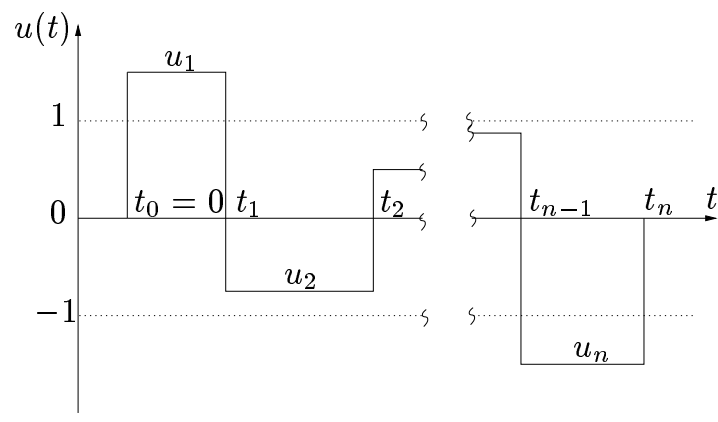

Figure 1: Control sequence.

solution of the linear system for $t \geq t_{0}=0$

$$
z(t)=e^{A t}\left(z(0)+\int_{0}^{t} e^{-A \tau} b v(\tau) d \tau\right)
$$

it is seen that that a control defined by the pair $(x, u)$ will steer $z_{0}$ to $z=0$ if it satisfies the 'steering equation'

$$
\Phi(x) u=-z_{0}
$$

where the $i$-th column of the matrix $\Phi$ is

$$
\Phi_{(:, i)}(x) \triangleq \int_{t_{i-1}}^{t_{i}} e^{-A \tau} b d \tau=\int_{\sum_{k=0}^{i-1} x_{k}}^{\sum_{k=0}^{i} x_{k}} e^{-A \tau} b d \tau
$$

The optimal vector $\bar{x}$ is the unique solution of (2.3) when $u=\bar{u}$ is the vector of optimal control values, that is $\left|\bar{u}_{i}\right|=1$ and $u_{i} u_{i+1}=-1$ for $i=1, \ldots, n-1$. The equation $\Phi(x) \bar{u}=-z_{0}$ is the nonlinear equation to be solved to determine the optimal control. In contrast, (2.3) is linear in $u$ and is easily solved for a given $x$. Because the pair $(A, b)$ is controllable, a unique solution $u(x)$ exists for any $x$ in the open positive orthant. A natural class of iterative methods thus consists in updating the time intervals vector $x$ such as to enforce convergence of the corresponding control vector $u(x)$ to a bang-bang sequence of magnitude $\left|u_{i}\right|=1$.

The heuristics considered in [15]-[16] and more recently in [2] is a "decentralized" adaptation of the vector $x$ : if $\left|u_{i}(x)\right|$ is larger than one, increase the length of the corresponding time interval $x_{i}$; if $\left|u_{i}(x)\right|$ is smaller than one, decrease the length of the corresponding time interval $x_{i}$.

In continuous-time, this heuristics yields the decentralized adaptation

$$
\dot{x}_{i}=f_{i}\left(\left|u_{i}(x)\right|-1\right) x_{i}, i=1, \ldots, n
$$

where $f_{i}$ should be a (smooth) scalar function in the first and third quadrant and only vanish at zero, and where $x_{i}$ is put in factor of $f_{i}$ in order to guarantee the positive invariance of the open positive orthant. If $z_{0}$ is not on a switching surface of the time-optimal control, the unique equilibrium of $(2.5)$ in $\mathcal{O}_{n}^{+}$is $\bar{x}$, the vector of optimal time intervals.

In discrete-time, the same heuristics yields the decentralized adaptation

$$
x_{i}(k+1)=x_{i}(k)+f_{i}\left(\left|u_{i}(x(k))\right|-1\right) x_{i}(k), i=1, \ldots, n
$$

which is a Euler discretization of (2.5).

In [16], $f_{i}$ is chosen as

$$
\begin{aligned}
f_{i}\left(\left|u_{i}\right|-1\right)= & q \ln \left|u_{i}\right| & \text { if } & \left|u_{i}(k)\right| \geq 1 \\
& -q \ln \left(2-\left|u_{i}\right|\right) & \text { if } & \left|u_{i}(k)\right|<1
\end{aligned}
$$

where $q>0$ is a constant parameter. In [2], $f_{i}$ is chosen as $f_{i}\left(\left|u_{i}\right|-1\right)=\left|u_{i}\right|^{p}-1$, with $0<p<1$ a constant parameter. Both algorithms were derived independently 
but it is apparent that they are closely related. Simulation studies in [2] show that both algorithms perform similarly, provided that the respective tuning parameters $p$ and $q$, are properly chosen. Several examples are included in [15]-[16], in which the algorithm is compared with other existing methods $[14,5,4]$. Extensive simulations suggest that both the continuous-time algorithm (2.5) and its discrete-time version (2.6) converge (globally) to the time-optimal solution and that the convergence properties are not very sensitive to the details of the function $f_{i}$, except for its slope at the origin (a higher slope seems to be favorable to the convergence).

The present paper provides a global analysis of the continuous-time system (2.5) with the functions $f_{i}$ selected as saturated linear functions, yielding the algorithm:

$\epsilon_{i} \dot{x}_{i}=\operatorname{sat}_{M}\left(\left|u_{i}(x)\right|-1\right) x_{i}, \quad i \in\{1, \cdots, n\}, x_{i}(0)>0$

where $\operatorname{sat}_{M}(y)=\operatorname{sign}(y) \min (M, y)$. With $0<\epsilon_{n}<<$ $\epsilon_{n-1}<<\cdots<<\epsilon_{1}$, a time-scale separation can be enforced between the different $x_{i}$ dynamics, and the different control values $\left|u_{i}\right|$ successively converge to 1 (starting with $\left|u_{n}\right|$ ).

\section{An example}

For the double integrator

$$
\left\{\begin{array}{l}
\dot{z}_{1}=z_{2} \\
\dot{z}_{2}=v
\end{array} \quad|v| \leq 1, \quad z(0)=\left(\begin{array}{c}
-1 \\
2
\end{array}\right)\right.
$$

the dynamical system (2.8) takes the form

$$
\left\{\begin{array}{lll}
\dot{x}_{1} & = & \operatorname{sat}\left(\left|u_{1}\right|-1\right) x_{1} \\
\epsilon \dot{x}_{2} & = & \operatorname{sat}\left(\left|u_{2}\right|-1\right) x_{2}
\end{array}\right.
$$

with $u=\left(u_{1}, u_{2}\right)^{T}$ solution of the steering equation:

$$
\left(\begin{array}{cc}
-\frac{x_{1}^{2}}{2} & -\frac{\left(x_{1}^{2}+x_{2}^{2}\right)-x_{1}^{2}}{2} \\
x_{1} & x_{2}
\end{array}\right)\left(\begin{array}{l}
u_{1} \\
u_{2}
\end{array}\right)=-\left(\begin{array}{l}
z_{1}(0) \\
z_{2}(0)
\end{array}\right)
$$

The phase portrait of (3.9) is illustrated on Figure 2 for $\epsilon$ very small. Fast convergence to the slow manifold $\left|u_{2}\right| \approx 1$ is followed by slow convergence to the equilibrium (indicated by a circle).

The manifold $\left|u_{2}\right|=1$ has two branches: the branch $u_{2}=+1$ goes through the equilibrium but exists only for $x_{1}>1$. The branch $u_{2}=-1$ only exists for $x_{1} \in$
$(0,1)$. The two branches merge at $\left(x_{1}, x_{2}\right)=(1,0)$, on the boundary of the positive orthant.

If the initial condition of (3.9) is chosen such that $x_{1} \in$ $(0,1)$, the solution first converges to the manifold $u_{2}=$ -1 . In the phase plane of the original system, this corresponds to a steering control of the form $\left(u_{1}, u_{2}\right)=$ $(-\hat{u},-1)$ where $\hat{u}$ is a positive number. The solution of (3.9) then "slides" along the manifold $u_{2}=-1$ up to the singular point $\left(x_{1}, x_{2}\right)=(1,0)$ before jumping to the manifold $u_{2}=+1$ where it is attracted to the equilibrium.

The next sections show that the behavior displayed in the above example generalizes to higher dimensions.

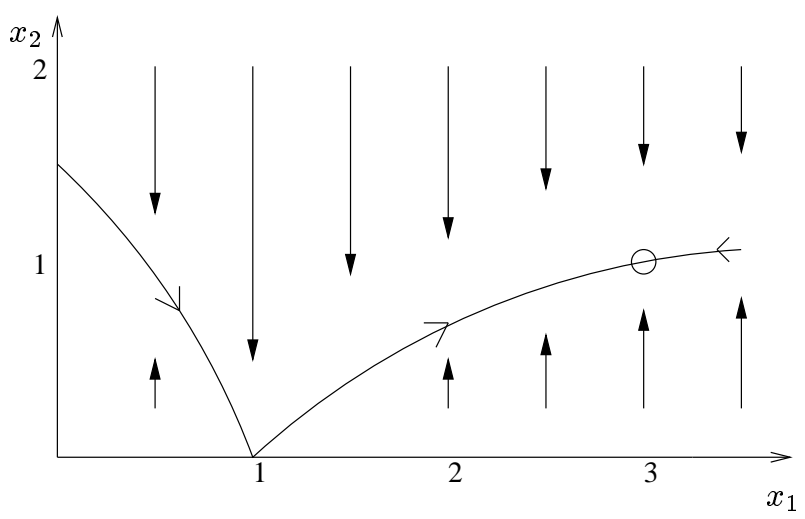

Figure 2: Phase portrait of (3.9) for $\epsilon$ small.

\section{Fast dynamics equilibria}

In the time-scale $\tau_{i}=\frac{t}{\epsilon_{i}}$, the fast dynamics of (2.8) are

$$
\begin{cases}\frac{\epsilon_{i+1}}{\epsilon_{i}} \dot{x}_{i+1} & =\operatorname{sat}\left(\left|u_{i+1}(x)\right|-1\right) x_{i+1} \\ & \vdots \\ \frac{\epsilon_{n}}{\epsilon_{i}} \dot{x}_{n} & =\operatorname{sat}\left(\left|u_{n}(x)\right|-1\right) x_{n}\end{cases}
$$

with $\left(x_{1}, \cdots, x_{i}\right)$ fixed. The slow dynamics of (2.8) then depend on the equilibria of (4.10). We will show that these equilibria are solutions of the following timeoptimal control problem:

$$
\begin{aligned}
\min T & =x_{i+1}+\cdots+x_{n} \\
\text { s.t. } & \dot{z}=A z+b v \quad|v| \leq 1 \\
& z(0) \in \Pi_{i}=e^{A t_{i}}\left(z_{0}+\operatorname{span}\left\{\Phi_{(:, 1)}, \cdots, \Phi_{(:, i)}\right\}\right) \\
& z(T)=0 \quad\left(\mathcal{P}_{i}\right)
\end{aligned}
$$

Note that $\mathcal{P}_{0}$ is the original optimal control problem considered in this paper, while $\mathcal{P}_{i}, 1 \leq i \leq n-1$, is the time-optimal control problem of reaching the origin $z=0$ from the affine subspace $\Pi_{i}$. The solution to this problem is unique and bang-bang. Regular solutions (for generic $\left.\left(x_{1}, \cdots, x_{i}\right)\right)$ involve $n-i-1$ switches 
and determine a unique sequence of switching intervals $\Omega\left(\mathcal{P}_{i}\right)=\left\{\left(x_{i+1}, \cdots, x_{n}\right)\right\}$, with the corresponding control sequence $\left(\tilde{u}_{i+1}, \cdots, \tilde{u}_{n}\right)$ alternating between +1 and -1 [7]. Singular solutions involve less than $n-i-1$ switches. In this case, the sequence of optimal switching intervals is not determined by a unique $\left(x_{i+1}, \cdots, x_{n}\right)$ of $\mathbb{R}^{n-i}$. For instance, if $n=2$, a singular steering control $\left\{\left(\begin{array}{l}\bar{x} \\ 0\end{array}\right),\left(\begin{array}{c}-1 \\ \star\end{array}\right)\right\}$ is equally represented by $\left\{\left(\begin{array}{l}\bar{x}_{1} \\ \bar{x}_{2}\end{array}\right),\left(\begin{array}{l}-1 \\ -1\end{array}\right)\right\}$ provided that $\bar{x}_{1}+\bar{x}_{2}=\bar{x}$. The set of vectors $\left(x_{i+1}, \cdots, x_{n}\right)^{T}$ that represent the same singular solution is denoted by $\Omega\left(\mathcal{P}_{i}\right)$; i.e.

$$
\begin{aligned}
& \Omega\left(\mathcal{P}_{i}\right)=\left\{\left(x_{i+1}, \cdots, x_{n}\right)^{T} \in \mathcal{O}_{n-i}^{+}: \exists\left(u_{i+1}, \cdots, u_{n}\right)^{T}\right. \\
& \quad \text { such that }\left\{\left(x_{i+1}, \cdots, x_{n}\right)^{T},\left(u_{i+1}, \cdots, u_{n}\right)^{T}\right\} \\
& \left.\quad \text { is solution of } \mathcal{P}_{i}\right\}
\end{aligned}
$$

When $\Omega\left(\mathcal{P}_{i}\right)$ is a singleton, we call the vector $\left(x_{1}, \cdots, x_{i}\right)^{T}$ regular. Otherwise, we call the vector $\left(x_{1}, \cdots, x_{i}\right)^{T}$ singular.

Lemma 1 Let $\left(x_{1}, \cdots, x_{i}\right)$ be a singular point.

Then, either $\Phi\left(x_{1}, \cdots, x_{i}, 0, \cdots, 0\right) u=-z_{0}$ has a solution, in which case, we call $\left(x_{1}, \cdots, x_{i}\right)$ a singularity of order $n-i$,

or there exists an integer $k, 1 \leq k<n-i$, and a unique $\left(x_{i+1}^{0}, \cdots, x_{n-k}^{0}\right)>0$ such that $\left|u_{j}\right|=1$ for $j \in\{i+1, \cdots, n-k\}$ and $u_{j} u_{j+1}=-1$ for $j \in\{i+1, \cdots, n-k-1\}$, and

$$
\Phi\left(x_{1}, \cdots, x_{i}, x_{i+1}^{0}, \cdots, x_{n-k}^{0}, 0, \cdots, 0\right) u=-z_{0}
$$

In the latter case, we call $\left(x_{1}, \cdots, x_{i}\right)$ a singularity of order $k$.

The proof of Lemma 1 follows from the fact that the optimal solution of $\mathcal{P}_{i}$ is bang-bang and unique. All the elements of $\Omega\left(\mathcal{P}_{i}\right)$ lead to steering controls that are equivalent to the steering control defined in Lemma 1.

Proposition 1 Let $\Omega\left(\mathcal{P}_{i}\right)$ be defined by (4.11) for a fixed $\left(x_{1}, \cdots, x_{i}\right)$.

(i) $\Omega\left(\mathcal{P}_{i}\right)$ characterizes the equilibrium set of (4.10) in $\mathcal{O}_{n-i}^{+}$.

(ii) Fix $\left(\bar{x}_{1}, \cdots, \bar{x}_{i-1}\right)>0$. Then for each $x_{i}, u_{i}(x)$, solution of $\Phi(x) u=-z_{0}$, is identical for all $\left(x_{i+1}, \cdots, x_{n}\right) \in \Omega\left(\mathcal{P}_{i}\right)$, i.e.

$$
u_{i}\left(\bar{x}_{1}, \cdots, \bar{x}_{i-1}, x_{i}, x_{i+1}, \cdots, x_{n}\right)=\hat{u}_{i}\left(x_{i}\right)
$$

(iii) $\hat{u}_{i}$ is continuous on $(0,+\infty)$

\section{Proof:}

(i) $\left(x_{i+1}, \cdots, x_{n}\right)$ is an equilibrium of $(4.10)$ in $\mathcal{O}_{n-i}^{+}$ if and only if $\left|u_{k}\right|=1$ for all $k \in\{i+1, \cdots, n\}$. The resulting steering control switches at most $n-i-1$ times. By uniqueness of the solution of $\mathcal{P}_{i}$, the steering control is optimal and $\left(x_{i+1}, \cdots, x_{n}\right) \in \Omega\left(\mathcal{P}_{i}\right)$

(ii) Because all points of $\Omega\left(\mathcal{P}_{i}\right)$ represent the same steering control, the impact point $z^{i}$ in $\Pi_{i}$ is identical for all $\left(x_{i+1}, \cdots, x_{n}\right) \in \Omega\left(\mathcal{P}_{i}\right)$. Because $\left(u_{1}, \cdots, u_{i}\right)$ is determined by the steering equation

$$
e^{A t_{i}} z_{0}+\sum_{j=1}^{i} e^{A t_{i}} \Phi_{(:, i)} u_{j}=z^{i}
$$

it follows that $u_{i}\left(\bar{x}_{1}, \cdots, \bar{x}_{i-1}, x_{i}, x_{i+1}, \cdots, x_{n}\right)$ is identical for all elements $\left(x_{i+1}, \cdots, x_{n}\right) \in \Omega\left(\mathcal{P}_{i}\right)$. Define $\hat{u}_{i}\left(x_{i}\right)=u_{i}\left(\bar{x}_{1}, \cdots, \bar{x}_{i-1}, x_{i}, x_{i+1}, \cdots, x_{n}\right)$ with $\left(x_{i+1}, \cdots, x_{n}\right) \in \Omega\left(\mathcal{P}_{i}\right)$.

(iii) The solution $\left(u_{1}, \cdots, u_{i}\right)$ of (4.12) is a continuous function of $z^{i}$. It is then a continuous function of $x_{i}$ if $z^{i}$ is a continuous function of $x_{i}$. It is shown in [10] (Sec. 2.5, Theorem 22) that the optimal time and the optimal control are continuous with respect to the initial condition. The initial set is here continuous with respect to $x_{i}$; therefore, the optimal time and the control law are continuous with respect to $x_{i}$. Continuity of the initial point $z^{i}$ with respect to $x_{i}$ follows directly.

\section{Slow dynamics analysis}

Fast convergence of the solutions of (4.10) to $\Omega\left(\mathcal{P}_{i}\right)$ will be proven in Section 6 . In the time-scale $\tau_{i}=\frac{t}{\epsilon_{i}}$, the slow dynamics of (2.8) then reduces to the scalar equation:

$$
\dot{x}_{i}=\operatorname{sat}\left(\left|\hat{u}_{i}\left(x_{i}\right)\right|-1\right) x_{i}
$$

with the continuous function $\hat{u}_{i}\left(x_{i}\right)$ determined as in Proposition 1.

Proposition 2 Denote by $\Gamma_{i}$ the equilibrium set of (5.13) in $(0, \infty)$.

- If $\left(x_{1}, \cdots, x_{i-1}\right)$ is regular, then $\Gamma_{i}=\left\{\bar{x}_{i}\right\}$ where $\bar{x}_{i}$ is uniquely determined by the solution of $\left(P_{i-1}\right)$.

- If $\left(x_{1}, \cdots, x_{i-1}\right)$ is singular of order $k(1 \leq k<$ $n-i+1)$, then $\Gamma_{i}=\left(0, x_{i}^{0}\right]$, where $x_{i}^{0}$ is determined by Lemma 1. 
- If $\left(x_{1}, \cdots, x_{i-1}\right)$ is singular of order $n-i+1$, then $\hat{u}_{i}\left(x_{i}\right) \equiv 0$, and $\Gamma_{i}=\emptyset\left(x_{i}=0\right.$ is the unique attractor of (5.13) which reduces to $\dot{x}_{i}=-x_{i}$ ).

The remainder of the section is devoted to the stability analysis of $\Gamma_{i}$. If $\left(x_{1}, \cdots, x_{i-1}\right)$ is regular, global attractivity of the equilibrium $\bar{x}_{i}$ is a consequence of the following result.

Proposition 3 If $\left(x_{1}, \cdots, x_{i}\right)$ is regular, then

$$
\frac{d \hat{u}_{i}}{d x_{i}}\left(x_{i}\right)\left(\hat{u}_{i}\left(x_{i}\right)-\tilde{u}_{i+1}\right)<0
$$

where the sign of $\tilde{u}_{i+1}= \pm 1$ is determined by the optimal solution of $P_{i}$

Proof: See [8].

Corollary 1 If $\left(x_{1}, \cdots, x_{i-1}\right)$ is regular, the unique equilibrium $\bar{x}_{i}$ of (5.13) is locally exponentially stable and globally attractive in $(0,+\infty)$.

Proof: Linearization of (5.13) at $x_{i}=\bar{x}_{i}$ yields

$$
\delta \dot{x}_{i}=\frac{d \hat{u}_{i}}{d x_{i}}\left(\bar{x}_{i}\right) \operatorname{sign}\left(\hat{u}_{i}\left(\bar{x}_{i}\right)\right) \bar{x}_{i} \delta x_{i}
$$

which is stable if $\frac{d \hat{u}_{i}}{d x_{i}} \operatorname{sign}\left(\hat{u}_{i}\left(x_{i}\right)\right)<0$. Noting that $\tilde{u}_{i}=$ $-\operatorname{sign}\left(\hat{u}_{i}\right)$ at the equilibrium, (5.14) becomes

$$
2 \frac{d \hat{u}_{i}}{d x_{i}} \operatorname{sign}\left(\hat{u}_{i}\left(x_{i}\right)\right)<0
$$

and local exponential stability is proven. For a scalar system with a single equilibrium, global attractivity follows from local stability.

To prove asymptotic stability of the equilibrium set $\left[0, x_{i}^{0}\right]$ when $\left(x_{1}, \cdots, x_{i-1}\right)$ is singular, we need the following proposition

Proposition 4 If $\left(x_{1}, \cdots, x_{i-1}\right)$ is regular (resp. singular of order smaller than $n-i+1)$, the values $x_{i}>0$ (resp. $\left.x_{i}>x_{i}^{0}\right)$ such that $\left(x_{1}, \cdots, x_{i}\right)$ is singular are isolated.

\section{Proof: See [8].}

Corollary 2 If $\left(x_{1}, \cdots, x_{i-1}\right)$ is singular of order $<$ $n-i+1$, the equilibrium set $\Gamma_{i}=\left(0, x_{i}^{0}\right]$ is globally asymptotically stable in $(0,+\infty)$.
Proof: $\quad$ By Proposition 2, $\left|\hat{u}_{i}\right|=1$ only if $x_{i} \in$ $\left[0, x_{i}^{0}\right]$. By Proposition 4, there exists $\epsilon^{*}>0$ such that $\left(x_{1}, \cdots, x_{i-1}, x_{i}+\epsilon\right)$ is regular for all $0<\epsilon<\epsilon^{*}$. For $x_{i} \in I_{\epsilon^{*}}=\left(x_{i}^{0}, x_{i}^{0}+\epsilon^{*}\right)$, the optimal control value $\tilde{u}_{i+1}\left(x_{i}\right)= \pm 1$ is constant.

We prove that $\tilde{u}_{i+1}\left(x_{i}\right)=-\hat{u}_{i}\left(x_{i}^{0}\right)$ for $x_{i} \in I_{\epsilon^{*}}$. By contradiction, suppose that $\tilde{u}_{i+1}\left(x_{i}\right)=\hat{u}_{i}\left(x_{i}^{0}\right)$. If $\hat{u}_{i}\left(x_{i}\right)>\tilde{u}_{i+1}\left(x_{i}\right)$, (5.14) forces $\frac{d \hat{u}_{i}}{d x_{i}}<0$ in the whole interval $I_{\epsilon^{*}}$, which, by continuity of $\hat{u}_{i}\left(x_{i}\right)$, contradicts that $\hat{u}_{i}\left(x_{i}\right)>\tilde{u}_{i+1}\left(x_{i}\right)$. The same reasoning can be held for $\hat{u}_{i}\left(x_{i}\right)<\tilde{u}_{i+1}\left(x_{i}\right)$ inside the interval. The only possibility is then that $\hat{u}_{i}\left(x_{i}\right)=\hat{u}_{i}\left(x_{i}^{0}\right)$, which contradicts Proposition 2.

We conclude that $\tilde{u}_{i+1}\left(x_{i}\right)=-\hat{u}_{i}\left(x_{i}^{0}\right)$ and that $\left|\hat{u}_{i}\left(x_{i}\right)\right|<\left|\tilde{u}_{i+1}\left(x_{i}\right)\right|$ for $x_{i} \in I_{\epsilon^{*}}$. This implies $\left|\hat{u}_{i}\left(x_{i}\right)\right|<1$ for all $x_{i}>x_{i}^{0}$. The set $\Gamma_{i}$ is then globally asymptotically stable in $(0, \infty)$.

\section{Global convergence analysis}

This section contains the main result of the paper and its proof. It puts together the fast and slow dynamics analyses of the previous two sections.

Theorem 1 Let $1 \leq i \leq n$ and fix $\left(x_{1}, \cdots, x_{i-1}\right)>0$ if $i>1$

Then the equilibrium set $\Omega\left(\mathcal{P}_{i-1}\right)$ of

$$
\left\{\begin{array}{cc}
\epsilon_{i} \dot{x}_{i} & =\operatorname{sat}\left(\left|u_{i}(x)\right|-1\right) x_{i} \\
& \vdots \\
\frac{\epsilon_{n}}{\epsilon_{i}} \dot{x}_{n}= & \operatorname{sat}\left(\left|u_{n}(x)\right|-1\right) x_{n}
\end{array}\right.
$$

is asymptotically stable. It is exponentially stable if $\Omega\left(\mathcal{P}_{i-1}\right)$ is a singleton.

Moreover, the region of attraction of $\Omega\left(\mathcal{P}_{i-1}\right)$ in the positive orthant is enlarged at will by proper separation of the time-scales $\tau_{n}=\frac{t}{\epsilon_{n}}, \cdots, \tau_{i}=\frac{t}{\epsilon_{i}}$

Proof: The proof goes by induction:

Let $i=n$ If $\left(x_{i}, \cdots, x_{n-1}\right)$ is regular, then $u_{n}(x)=$ $\hat{u}_{n}\left(x_{n}\right)$ and the unique equilibrium $\bar{x}_{n}$ of $\dot{x}_{n}=$ $\operatorname{sat}\left(\left|\hat{u}_{n}\left(x_{n}\right)\right|-1\right) x_{n}$ is exponentially stable and globally attractive, see Corollary 1.

If $\left(x_{i}, \cdots, x_{n-1}\right)$ is singular, then $\Pi_{n-1}$ contains the origin $z=0$ and $u_{n}(x)=\hat{u}_{n}\left(x_{n}\right)=0$. Then $x_{n}=0$ is globally exponentially stable. 
Induction step Consider (6.15) with $1 \leq i<n$. Denote by $\Gamma_{i}$ the equilibrium set of

$$
\epsilon_{i} \dot{x}_{i}=\operatorname{sat}\left(\left|\hat{u}_{i}\left(x_{i}\right)\right|-1\right) x_{i}
$$

and by $\Sigma_{i}$ the subset of $\mathbb{R}_{0}^{+} \backslash \Gamma_{i}$ such that $x_{i} \in \Sigma_{i}$ implies that $\left(x_{1}, \cdots, x_{i}\right)$ is singular.

Assume first that $\Sigma_{i}=\emptyset$. Then the fast subsystem of (6.15) has a unique equilibrium for all $x_{i} \in \mathbb{R}_{0}^{+}$ $\Gamma_{i}$, and this equilibrium is exponentially stable with an arbitrarily large region of attraction from the induction hypothesis. As a consequence, there exists an invariant slow manifold in the neighborhood of $\mathbb{R}_{0}^{+} \backslash \Gamma_{i} \times \Omega\left(\mathcal{P}_{i}\right)$. On the other hand, the equilibrium set $\Gamma_{i}$ of (6.15) is globally asymptotically stable (see Corollaries 1 and 2).

From standard singular perturbations theory (e.g. Theorem 3.18 in [13]), the equilibrium set $\Gamma_{i} \times \Omega\left(\mathcal{P}_{i}\right)$ of (6.15) is locally asymptotically stable and its region of attraction can be made arbitrarily large be making $\frac{\epsilon_{i+1}}{\epsilon_{i}}$ small enough.

Next suppose that $\Sigma_{i} \neq \emptyset$. Denote by $\Sigma_{i}^{\gamma}$ a closed neighborhood of $\Sigma_{i}$ such that $\Sigma_{i}^{\gamma} \cap \Gamma_{i}=\emptyset$ and such that $\Sigma_{i}^{\gamma}$ is made of disjoint closed intervals $I_{l}^{\gamma}$ of length $\gamma(l \geq 1)$. By Proposition 4, $\gamma>0$ can be selected arbitrarily small. Consider a solution that satisfy $x_{i}(t) \in I_{l}^{\gamma}$ for all $t>0$ and let $\tilde{x}_{i}$ be a singularity of maximal order in $I_{l}^{\gamma}$. Then $\left(x_{i+1}(t), \cdots, x_{n}(t)\right)$ converges to a $\gamma$ neighborhood $\Omega^{\gamma}\left(\mathcal{P}_{i}\right)\left(\tilde{x}_{i}\right)$ of $\Omega\left(\mathcal{P}_{i}\right)\left(\tilde{x}_{i}\right)$. Because ||$\hat{u}_{i}\left(\tilde{x}_{i}\right)|-1| \geq$ $\delta$, for some $\delta>0$ and $u_{i}\left(x_{i}, \cdots, x_{n}\right)$ is continuous, there exists $\gamma$ small enough such that ||$u_{i}(\tilde{x})|-1| \geq \frac{\delta}{2}$ for all $x_{i} \in I_{l}^{\gamma}$ and $\left(x_{i+1}(t), \cdots, x_{n}(t)\right) \in \Omega^{\gamma}\left(\mathcal{P}_{i}\right)\left(\tilde{x}_{i}\right)$. But then $\dot{x}_{i}$ is sign definite for $t$ large enough and does not converge to zero, which contradicts the fact that $x_{i}(t) \in I_{l}^{\gamma}$ for all $t>0$. The slow dynamics (5.13) being valid in $\left(\mathbb{R}^{+} \backslash \Gamma_{i}\right) \cup \Sigma_{i}^{\gamma}$, we conclude that all solutions converge to $\Gamma_{i} \times \Omega\left(\mathcal{P}_{i}\right)$.

\section{Conclusion}

In this paper we have analyzed the global convergence properties of a continuous flow which computes the solution of a linear time-optimal control problem. The analysis rests on a time-scale separation of the dynamics into $n$ scalar dynamics, each of one characterizing the transfer from one hyperplane to a switching surface of the optimal control problem.

\section{References}

[1] Bellman, R., Glicksberg, I., Gross, O. (1956), "On the bang-bang control problem," Quarterly of Applied Mathematics, 14, 11-18.
[2] De Doná, J.A. (2000), "Input Constrained Linear Control," PhD. thesis, The University of Newcastle, Australia.

[3] Desoer, C.A. (1959), "The bang-bang servo problem treated by variational techniques," Information and Control, 2, pp. 333-348.

[4] Fadden, E.J. (1965), Computational aspects of a class of optimal control problems, Technical Report ORA Project 06181, University of Michigan, Ann Arbor.

[5] Fadden, E.J., Gilbert E.G. (1964), Computational aspects of the time optimal control problem, in A.V. Balakrishnan \& L.W. Neustadt, eds, "Computing Methods in Optimization Problems," Academic Press, New York.

[6] Gilbert E.G. (1964), "The application of hybrid computers to the iterative solution of optimal control problems, in A.V. Balakrishnan \& L.W. Neustadt, eds, "Computing Methods in Optimization Problems," Academic Press, New York, pp. 261-284.

[7] Grognard F., Sepulchre R., De Dona J. (2001), "Dynamical systems that compute timeoptimal switchings," NOLCOS 2001.

[8] Grognard F., Sepulchre R., "Global analysis of a continuous-time flow which computes time-optimal switchings," CESAME internal report No. 2001.18, Université Catholique de Louvain, Belgium.

[9] Ho, Y.C. (1962), "A successive approximation technique for optimal control systems subject to input saturation," Journal of Basic Engineering, Transactions of ASME, 84D, 33-40.

[10] Lee, E.B., Markus, L. (1967), "Foundations of Optimal Control Theory," John Wiley \& Sons, Inc., New York.

[11] Mayne, D.Q., Rawlings, J.B., Rao, C.V., Schockaert, P.O.M. (2000), "Constrained model predictive control: stability and optimality," Automatica, 36, pp. 789-814.

[12] Neustadt, L.W. (1960), "Synthesizing timeoptimal control systems," Journal of Mathematical Analysis and Applications, 1, pp. 464-493.

[13] R. Sepulchre, M. Jankovic, P.V. Kokotović, Constructive Nonlinear Control, Springer-Verlag, 1996.

[14] Smith, F.B. (1961), "Time-optimal control of higher-order systems," IRE Trans. on Automatic Control, 6, pp. 16-21.

[15] Yastreboff, M.M. (1969), "Investigation of Several Synthesis Procedures for On-Line Time Optimal Control," PhD. thesis, The University of New South Wales, Australia.

[16] Yastreboff, M.M. (1969), "Synthesis of timeoptimal control by time interval adjustment," IEEE Trans. on Automatic Control, 14, pp. 707-710 . 